To appear in Complex Variables and Elliptic Equations

Vol. 00, No. 00, Month 20XX, 1-19

\title{
On the completeness of root functions of a holomorphic family of non-coercive mixed problem
}

\author{
A.N. Polkovnikov ${ }^{\text {** }}$ \\ ${ }^{a}$ Institute of Mathematics and Computer Science, Siberian Federal University, Svobodny \\ Prospect 79, Krasnoyarsk, 660041, Russia
}

(Received 00 Month 20XX; final version received 00 Month 20XX)

\begin{abstract}
We consider a non-coercive mixed boundary value problem in a bounded domain $D$ of complex space $\mathbb{C}^{n}$ for a second order parameter-dependent elliptic differential operator $A(z, \bar{\partial}, \lambda)$ with complex-valued essentially bounded measured coefficients and complex parameter $\lambda$. The differential operator is assumed to be of divergent form in $D$, the boundary operator $B(z, \bar{\partial})$ is of Robin type. The boundary of $D$ is assumed to be a Lipschitz surface. Under reasonable assumptions the pair $(A, B)$ induces a family of non-coercive mixed problems and a holomorphic family of Fredholm operators $L(\lambda): H^{+}(D) \rightarrow H^{-}(D)$ in suitable Hilbert spaces $H^{+}(D) \subset H^{1 / 2}(D)$, $H^{-1 / 2}(D) \subset H^{-}(D)$ of Sobolev type (here $H^{s}(D)$ are the Sobolev-Slobodetskii spaces over $D$ ). If there is a Lipschitz function close enough to the (possibly discontinuous) argument of the complex-valued multiplier of the parameter $\lambda$ in $A(z, \bar{\partial}, \lambda)$ then we prove that the operators $L(\lambda)$ are continuously invertible for all $\lambda$ with sufficiently large modulus $|\lambda|$ on each angle on the complex plane $\mathbb{C}$ where the operator $A(z, \bar{\partial}, \lambda)$ is parameter-dependent elliptic. We also describe reasonable conditions for the system of root functions related to the family $L(\lambda)$ to be (doubly) complete in the spaces $H^{+}(D), H^{-}(D)$ and the Lebesgue space $L^{2}(D)$.
\end{abstract}

Keywords: parameter-depended elliptic operator, non-coercive problem, root functions, holomorphic family.

AMS Subject Classification: 35J25, 35P10

\section{Introduction}

It is well-known that the Shapiro-Lopatinsky conditions with parameter provide the coercive estimates for mixed boundary value problems for parameter-dependent elliptic operators, see, for instance, [1] (cf. also some recent generalizations in [2]). It is important at least for two reasons: first, because the notion of a parameterdependent elliptic operator provides a useful link between the theories of boundary value problems for parabolic and elliptic operators and second, because it provides a justification for application of Galerkin type methods and numerical solution of the problem in the case where one succeeds with finding an information on the completeness of the system of eigenfunctions related to the problem (see, for example, $[3],[4],[5],[6],[7]$ for the corresponding base fact in functional analysis and [8], $[9],[10],[11]$ for the realization for problems over smooth domains. It is worth to

* Corresponding author. Email: PaSkaattt@yandex.ru 
note that the spectral theory in non-smooth domains usually depends upon hard analysis near singularities on the boundary (see, for instance, [12], [13]).

On the other hand, the classical approach can be also adapted for investigation of non-coercive mixed problems over domains with Lipschitz boundaries (see [14] for the spectral problems and [15] for parameter dependent-elliptic operators). An essential part of the approach is the analysis in spaces of negative smoothness. We use this method to prove that under reasonable assumptions the non-coercive operator pencil $L(\lambda): H^{+}(D) \rightarrow H^{-}(D)$ has almost the same properties as a coercive one. Actually the present results are generalizations of the ones published in [15]. In contrast to [15] we do not assume the continuity of the argument of the complex-valued multiplier of the parameter in the parameter-dependent elliptic operator under the consideration.

\section{Mixed problem for parameter-depended elliptic operator}

Let $D$ be a bounded domain with a Lipschitz boundary in the complex space $\mathbb{C}^{n} \cong$ $\mathbb{R}^{2 n}$ with the coordinates $z_{j}=x_{j}+\sqrt{-1} x_{n+j}, j=1, \ldots, n, x \in \mathbb{R}^{2 n}$, i.e., the surface $\partial D$ is locally the graph of a Lipschitz function. As far as is known, the Lipschitz boundary $\partial D$ possesses a tangent hyperplane almost everywhere.

As usual we denote by $\bar{\partial}$ the Cauchy-Riemann operator in $\mathbb{C}^{n}$, i.e., the column of $n$ formal complex derivatives

$$
\bar{\partial}_{j}=\frac{\partial}{\partial \bar{z}_{j}}=\frac{1}{2}\left(\frac{\partial}{\partial x_{j}}+\sqrt{-1} \frac{\partial}{\partial x_{n+j}}\right), 1 \leq j \leq n .
$$

The formal adjoint $\bar{\partial}^{*}$ of Cauchy-Riemann operator is line of $n$ formal complex derivatives $\bar{\partial}_{j}^{*}=:-\frac{\partial}{\partial z_{j}}$, thus $\bar{\partial}_{j}^{*}=-\frac{1}{2}\left(\frac{\partial}{\partial x_{j}}-\sqrt{-1} \frac{\partial}{\partial x_{n+j}}\right)$.

We consider complex-valued functions defined in the domain $D \subset \mathbb{C}^{n}$ and its closure $\bar{D}$. Denote by $L^{q}(D)$ the Lebesgue space, i.e. the set of all measurable functions $u$ in $D$, such that the integral of $|u|^{q}$ over $D$ is finite. We also write $H^{s}(D), s \in \mathbb{N}$, for the corresponding Sobolev space of functions with all the weak derivatives up to order $s$ belonging to $L^{2}(D)$. For non-negative non-integer $s$ we denote by $H^{s}(D)$ the Sobolev-Slobodetskii space (see, for instance, [10]).

Consider a second order parameter-dependent partial differential operator $A(z, \bar{\partial}, \lambda)$ of a divergence form

$$
A(z, \bar{\partial}, \lambda) u=-\sum_{i, j=1}^{n} \bar{\partial}_{i}^{*}\left(a_{i, j}(z) \bar{\partial}_{j}\right) u+\sum_{j=1}^{n} a_{j}(z) \bar{\partial}_{j} u+a_{0}(z) u+\lambda^{2} a_{0}^{(2)}(z) u
$$

the coefficients $a_{i, j}(z), a_{j}(z), a_{0}(z), a_{0}^{(2)}(z)$ being bounded measurable complexvalued functions in $D$. Assuming that $a_{i, j}(z)$ are continuous up to the boundary of $D$ we can consider the first order Robin type boundary differential operator

$$
B(z, \bar{\partial}) u=b_{1}(z) \bar{\partial}_{\nu} u+b_{0} u
$$

where $\bar{\partial}_{\nu}=\sum_{j=1}^{n} a_{j, i}\left(\nu_{j}(x)-\sqrt{-1} \nu_{j+n}(x)\right) \frac{\partial}{\partial \bar{z}_{j}}$ is the complex conormal derivative and $\nu(x)=\left(\nu_{1}(x), \ldots \nu_{2 n}(x)\right)$ is the unit normal vector to $\partial D$ at the point $z$ (cf. 
with the usual normal derivative $\left.\frac{\partial}{\partial \nu}=\sum_{j=1}^{2 n} \nu_{j}(x) \frac{\partial}{\partial x_{j}}\right)$. The coefficients $b_{0}(z)$, $b_{1}(z)$ are assumed to be bounded measurable functions on $\partial D$. We also allow the function $b_{1}(z)$ to vanish on an open connected subset $S$ of $\partial D$ with piecewise smooth boundary $\partial S$, and we assume that $b_{0}(z) \neq 0$ for $z \in S$.

Denote by $H^{s}(D, S)$ the subspace of $H^{s}(D), s>1 / 2$, consisting of those functions whose restriction to the boundary vanishes on $S$. This is a Hilbert space under the induced norm. It is well-known that smooth functions on $\bar{D}$ vanishing in a neighborhood of $\bar{S}$ are dense in $H^{1}(D, S)$; then the space $H^{1}(D, \partial D)$ is usually denoted $H_{0}^{1}(D)$. Since on $S$ the boundary operator reduces to $b_{0}(z)$ and $b_{0}(z) \neq 0$ for $z \in S$, the functions of $H^{1}(D)$ satisfying $B(z, \bar{\partial}) u=0$ on $\partial D$ belong to $H^{1}(D, S)$.

We consider the following family of boundary value problems. Given data $f$ in $D$ and $u_{0}$ in $\partial D$, find a distribution $u$ in $\bar{D}$, which satisfies

$$
\begin{cases}A(z, \bar{\partial}, \lambda) u=f & \text { in } \quad D \\ B(z, \bar{\partial}) u=u_{0} & \text { on } \quad \partial D .\end{cases}
$$

To study the problem we have to put some restrictions on the operators $A(z, \bar{\partial}, \lambda)$ and $B(z, \bar{\partial})$. We suppose that, for each $z \in \mathbb{C}^{n}$, the matrix

$$
\left(a_{i, j}(z)\right)_{\substack{i=1, \ldots, n \\ j=1, \ldots, n}}
$$

is Hermitian and there is a constant $m>0$ such that

$$
\sum_{i, j=1}^{n} a_{i, j}(z) \bar{w}_{i} w_{j} \geq m|w|^{2}
$$

for all $(z, w) \in \bar{D} \times\left(\mathbb{C}^{n} \backslash\{0\}\right)$.

Split now both $a_{0}$ and $b_{0}$ into two parts

$$
\begin{aligned}
& a_{0}=a_{0,0}+\delta a_{0}, \\
& b_{0}=b_{0,0}+\delta b_{0},
\end{aligned}
$$

where $a_{0,0}$ is a non-negative bounded function in $D$ and $b_{0,0}$ is a bounded function on $\partial D$ satisfying $b_{0,0} / b_{1} \geq 0$. Then, under negligible restrictions, the following Hermitian form

$$
(u, v)_{+}=\sum_{i, j=1}^{n}\left(a_{i, j} \bar{\partial}_{j} u, \bar{\partial}_{i} v\right)_{L^{2}(D)}+\left(a_{0,0} u, v\right)_{L^{2}(D)}+\left(b_{0,0} b_{1}^{-1} u, v\right)_{L^{2}(\partial D \backslash S)}
$$

defines a scalar product on $H^{1}(D, S)$ (see [14]). Denote by $H^{+}(D)$ the completion of $H^{1}(D, S)$ with respect to the norm $\|\cdot\|_{+}$induced by the scalar product $(\cdot, \cdot)_{+}$. Estimate (2) implies that

$$
\|u\|_{+} \geq \sqrt{m}\|\bar{\partial} u\|_{L^{2}(D)}
$$


for all $u \in H^{1}(D, S)$; however it does not provide the coercive estimate

$$
\|u\|_{+} \geq c\|\nabla u\|_{L^{2}(D)}
$$

for all $u \in H^{1}(D, S)$ with a constant $c$ independent on $u$.

However the following embedding theorem holds for the space $H^{+}(D)$.

THEOREM 2.1 Let there be a constant $c_{1}>0$, such that

$$
\frac{b_{0,0}}{b_{1}} \geq c_{1} \text { on } \partial D \backslash S
$$

with any $\varepsilon>0$. If, in addition, $\partial D \in C^{2}$ then the space $H^{+}(D)$ is continuously embedded into $H^{1 / 2}(D)$.

Proof. According to [14, Theorem 2.5], for the space $\tilde{H}^{+}(D)$, defined as the completion of $H^{1}(D, S)$ with respect to the norm $\|\cdot\|_{\tilde{+}}$ coherent with the scalar product

$$
(\cdot, \cdot)_{\tilde{+}}=(\bar{\partial} u, \bar{\partial} v)_{L^{2}(D)}+\left(a_{0,0} u, v\right)_{L^{2}(D)}+\left(b_{0,0} b_{1}^{-1} u, v\right)_{L^{2}(\partial D \backslash S)},
$$

the statement of the theorem holds true. Using (4) we see that the norm $\|\cdot\|_{+}$is not weaker than $\|\cdot\|_{\tilde{+}}$, and the statements follows.

We note, that the space $H^{+}(D)$ can not be continuously embedded into $H^{1}(D)$ in our case. Indeed, as $a_{i, j} \in L^{\infty}(D)$ we see that there is a positive constant $c$ such that

$$
\sum_{i, j=1}^{n}\left(a_{i, j} \bar{\partial}_{j} u, \bar{\partial}_{i} u\right)_{L^{2}(D)} \leq c\|\bar{\partial} u\|_{L^{2}(D)}^{2}
$$

for all $u \in H^{1}(D, S)$. This means that the norms $\|\cdot\|_{+}$and $\|\cdot\|_{\tilde{r}}$ are equivalent. According to [14, Remark 5.1] $\tilde{H}^{+}(D)$ can not be continuously embedded into $H^{1}(D)$. Thus Hermitian form (3) is non-coercive.

Let now $H^{-}(D)$ be the dual space for the space $H^{+}(D)$ with respect to the pairing $\langle\cdot, \cdot\rangle$ induced by the scalar product $(\cdot, \cdot)_{L^{2}(D)}$, see [10] and elsewhere. More precisely, let $H^{-}(D)$ be the completion of $H^{+}(D)$ with respect to the negative norm

$$
\|u\|_{-}=\sup _{\substack{v \in H^{+}(D) \\ v \neq 0}} \frac{\left|(v, u)_{L^{2}(D)}\right|}{\|v\|_{+}} .
$$

Then an integration by parts leads to a weak formulation of problem (1): given $f \in H^{-}(D)$, find $u \in H^{+}(D)$, such that

$$
(u, v)_{+}+\left(\left(\sum_{j=1}^{n} a_{j} \frac{\partial}{\partial \bar{z}_{j}}+\delta a_{0}+\lambda^{2} a_{0}^{(2)}\right) u, v\right)_{L^{2}(D)}+\left(b_{1}^{-1} \delta b_{0} u, v\right)_{L^{2}(\partial D \backslash S)}=<f, v>
$$

for all $v \in H^{+}(D)$. Note, that (6) induces a holomorphic (with respect to the $\lambda$ ) 
family $L(\lambda): H^{+}(D) \rightarrow H^{-}(D), \lambda \in \mathbb{C}$, of bounded linear operators, if

$$
\left|\left(b_{1}^{-1} \delta b_{0} u, v\right)_{L^{2}(\partial D \backslash S)}\right| \leq c\|u\|_{+}\|v\|_{+}
$$

with a positive constant $c>0$ for all $u, v \in H^{+}(D)$.

The bounded linear operator $L_{0}: H^{+}(D) \rightarrow H^{-}(D)$ defined in the same way via the sesquilinear form $(\cdot, \cdot)_{+}$,

$$
(u, v)_{+}=\left\langle L_{0} u, v\right\rangle
$$

for all $u, v \in H^{+}(D)$, corresponds to the case $\lambda=0, a_{j} \equiv 0$ for all $j=1, \ldots, n$, $a_{0}=a_{0,0}$ and $b_{0}=b_{0,0}$. Clearly (see for instance [14, Lemma 2.6]), the operator $L_{0}: H^{+}(D) \rightarrow H^{-}(D)$ is continuously invertible and $\left\|L_{0}\right\|=\left\|L_{0}^{-1}\right\|=1$.

Consider the sesquilinear form on $H^{-}(D)$ given by

$$
(u, v)_{-}:=\left\langle L_{0}^{-1} u, v\right\rangle
$$

for all $u, v \in H^{-}(D)$. This form defines the scalar product on $H^{-}(D)$, which is coherent with the norm $\|\cdot\|_{-}$(see, for instance [14]).

LEMma 2.2 Let the hypothesis of Theorem 2.1 be fulfilled and (7) hold true with some constant $c<1$. Then $\{L(\lambda)\}_{\lambda \in \mathbb{C}}$ is a holomorphic family of Fredholm operators of zero index.

Proof. Under conditions of the lemma, Rellich Theorem and Theorem 2.1 imply that $H^{+}(D)$ is compactly embedded into $L^{2}(D)$. Therefore (see for instance [14, Lemma 2.2]), $L^{2}(D)$ is compactly embedded into $H^{-}(D)$.

It follows that operator $\delta L(\lambda)=\left(\delta_{c} L+\lambda^{2} C\right): H^{+}(D) \rightarrow H^{-}(D)$, induced by the summand $\left(\delta a_{0}+\sum_{j=1}^{n} a_{j} \frac{\partial}{\partial \bar{z}_{j}}+\lambda^{2} a_{0}^{(2)}\right)$, is compact. Indeed, the operator $\left(\delta a_{0}+\sum_{j=1}^{n} a_{j} \frac{\partial}{\partial \bar{z}_{j}}+\lambda^{2} a_{0}^{(2)}\right)$ continuously maps the space $H^{+}(D)$ into $L^{2}(D)$, because $a_{0}, a_{j}, a_{0}^{(2)} \in L^{\infty}(D)$ for all $j=1, \ldots, n$, and $\lambda$ is a constant. Since $L^{2}(D)$ is compactly embedded into $H^{-}(D)$, the operator $\delta L(\lambda)$ is compact.

Further, denote by $\delta_{b} L: H^{+}(D) \rightarrow H^{-}(D)$ the operator, induced by the term $b_{1}^{-1} \delta b_{0}$. Under the hypothesis of the Lemma it follows, that $\left\|\delta_{b} L\right\|<1$. Since operator $L_{0}$ is continuously invertible and $\left\|L_{0}\right\|=1$, the operator $L_{0}+\delta_{b} L$ is continuously invertible. So we conclude that for each $\lambda \in \mathbb{C}$ the operator $L(\lambda)=L_{0}+\delta_{b} L+\delta_{c} L+\lambda^{2} C$ is Fredholm. Thus we obtain the holomorphic family $\{L(\lambda)\}_{\lambda \in \mathbb{C}}$ of Fredholm operators of zero index.

Consider now the operator $A(z, \bar{\partial}, \lambda)$. We suppose that $A(z, \bar{\partial}, \lambda)$ is a parameterdepended elliptic operator in an angle $K=\left\{\alpha \leq \varphi_{\lambda} \leq \beta\right\}$ on a complex plane $\mathbb{C}$, i.e.

$$
\sum_{i, j=1}^{n} a_{i, j}(z) \xi_{i} \xi_{j}+\lambda^{2} a_{0}^{(2)}(z) \neq 0
$$

for all $(z, \xi, \lambda) \in \bar{D} \times\left[\left(\mathbb{R}^{n} \times K\right) \backslash\{(0,0)\}\right]$, where $\varphi_{\lambda}=\arg (\lambda)$ and $\alpha, \beta$ some constant, $0 \leq \alpha \leq \beta<2 \pi$. It is clear that $K$ is a ray if $\alpha=\beta$. 
LEMMA 2.3 Let the matrix $\left(a_{i, j}(z)\right)_{\substack{i=1, \cdots, n \\ j=1, \cdots, n}}$ be Hermitian and (2) be fulfilled. Then $A(z, \bar{\partial}, \lambda)$ is a parameter-depended elliptic operator in the angle $K$ if and only if

$$
\begin{gathered}
\left|a_{0}^{(2)}(z)\right|>0 \text { for all } z \in \bar{D} \\
\cos \left(\varphi_{0}(z)+2 \varphi_{\lambda}\right)>-1 \text { for all } z \in \bar{D} \text { and } \alpha \leq \varphi_{\lambda} \leq \beta ;
\end{gathered}
$$

where $\varphi_{0}(z)=\arg \left(a_{0}^{(2)}(z)\right)$.

Proof. Let (10) holds true, if we take $\xi=0$ then $a_{0}^{(2)}(z) \neq 0$ for all $z \in \bar{D}$, in particular (11) is fulfilled. If $\lambda \neq 0$, as $a_{0}^{(2)}(z) \neq 0$ for all $z \in \bar{D}$ and (2) is fulfilled, then

$$
|\lambda|^{2}\left|a_{0}^{(2)}(z)\right|\left(\cos \left(\varphi_{0}(z)+2 \varphi_{\lambda}\right)+\sqrt{-1} \sin \left(\varphi_{0}(z)+2 \varphi_{\lambda}\right)\right)+\sum_{i, j=1}^{n} a_{i, j}(z) \xi_{i} \xi_{j}=0
$$

if and only if the following two conditions hold:

$$
\left\{\begin{array}{l}
\sin \left(\varphi_{0}(z)+2 \varphi_{\lambda}\right)=0 \\
\cos \left(\varphi_{0}(z)+2 \varphi_{\lambda}\right) \leq 0
\end{array}\right.
$$

which are fulfilled if and only if $\cos \left(\varphi_{0}(z)+2 \varphi_{\lambda}\right)=-1$ for all $z \in \bar{D}$ and $\alpha \leq \varphi_{\lambda} \leq \beta$. Therefore (10) implies (12), too.

On the other hand, let (10) be not true for a triple $\left(z_{0}, \xi_{0}, \lambda_{0}\right) \in \bar{D} \times$ $\left[\left(\mathbb{C}^{n} \times K\right) \backslash\{(0,0)\}\right]$. Then $\lambda_{0} \neq 0$ because of (2). If $\xi_{0}=0$ then (13) implies $\left|a_{0}^{(2)}\left(z_{0}\right)\right|=0$. Finally, if $\xi_{0} \neq 0$, it follows from (2), (13) and (14) that

$$
\left|a_{0}^{(2)}(z)\right|>0 \text { and } \cos \left(\varphi_{0}(z)+2 \varphi_{\lambda}\right)=-1,
$$

i.e. or (11) does not hold or (12) is not fulfilled at the point $z_{0} \in \bar{D}$.

If $\left|a_{0}^{(2)}(z)\right| \in C(\bar{D})$ then in our case (11) is equivalent to the following

$$
\left|a_{0}^{(2)}(z)\right| \geq \theta_{0}>0 \text { for all } z \in \bar{D}
$$

similarly, if $\varphi_{0}(z) \in C(\bar{D})$ then in our case (12) is equivalent to the following

$$
\cos \left(\varphi_{0}(z)+2 \varphi_{\lambda}\right) \geq \theta_{1}>-1 \text { for all } z \in \bar{D}, \varphi_{\lambda} \in K
$$

where the constants $\theta_{0}, \theta_{1}$ do not depend on $z$. It is easy to see that operator $C$ is invertible, if

$$
a_{0}^{(2)}(z) \neq 0 \text { for almost all } z \in D .
$$

Lemma 2.4 Under the hypothesis of Lemma 2.2, if there is $\lambda_{0} \in \mathbb{C}$ such that $L\left(\lambda_{0}\right)$ is continuously invertible, then the holomorphic family of bounded linear operators 
$L(\lambda): H^{+}(D) \rightarrow H^{-}(D)$ are continuously invertible for all $\lambda \in \mathbb{C}$ except a countable sequence $\left\{\lambda_{\nu}\right\}$ of isolated points on the complex plane.

Proof. Under the hypothesis of Lemma 2.2, the holomorphic (polynomial) family

$$
\left\{L(\lambda)=L(0)+\lambda^{2} C\right\}
$$

consists of Fredholm operators of zero index. Clearly

$$
L(\lambda)=L\left(\lambda_{0}\right)+\left(\lambda^{2}-\lambda_{0}^{2}\right) C .
$$

As $L\left(\lambda_{0}\right)$ is continuously invertible we conclude that the operator $L\left(\lambda_{0}\right)$ is continuously invertible if and only if the operator

$$
T(\lambda)=I+\left(\lambda^{2}-\lambda_{0}^{2}\right) L^{-1}\left(\lambda_{0}\right) C
$$

has this property. Since the operator $C$ is compact and the operator-valued function

$$
h(\lambda)=\left(\lambda^{2}-\lambda_{0}^{2}\right) L^{-1}\left(\lambda_{0}\right) C
$$

is holomorphic in $\mathbb{C}$, vanishing at the point $\lambda_{0}$, it follows from ([4, Ch. I, Theorem $5.1]$ ), that $T(\lambda)$ is continuously invertible for all $\lambda \in \mathbb{C}$ except a countable sequence $\left\{\lambda_{\nu}\right\}$ of isolated singular points on the complex plane.

The next theorem is an analogue of [15, Theorem 3] with discontinuous argument of function $a_{0}^{(2)}(z)$.

TheOREM 2.5 Let the hypothesis of Lemma 2.4 be fulfilled and (17), (18) hold true. If either $\theta_{1} \geq 0$ and $\left\|\delta_{b} L\right\|<1$ or $\theta_{1}<0$ and there exist a function $\varphi_{0,0}(z) \in C^{0,1}(\bar{D})$ such that

$$
\left\|1-e^{\sqrt{-1}\left(\varphi_{0}-\varphi_{0,0}\right)}\right\|_{L^{\infty}(D)}<1-\left|\theta_{1}\right|
$$

and $\left\|\delta_{b} L\right\|<\sqrt{1-\left(\left|\theta_{1}\right| /\left(1-\left\|1-e^{\sqrt{-1}\left(\varphi_{0}-\varphi_{0,0}\right)}\right\|_{L^{\infty}(D)}\right)\right)^{2}}$ then

1) there is $\lambda_{0} \in K$ such that the operators $L(\lambda): H^{+}(D) \rightarrow H^{-}(D)$ are continuously invertible for all $\lambda \in K$ with $|\lambda| \geq\left|\lambda_{0}\right|$;

2) the operators $L(\lambda)$ are continuously invertible for all $\lambda \in \mathbb{C}$ except a discrete countable set $\left\{\lambda_{\nu}\right\}$ without limit points in $\mathbb{C}$.

Proof. Let $d=1-\left\|1-e^{\sqrt{-1}\left(\varphi_{0}-\varphi_{0,0}\right)}\right\|_{L^{\infty}(D)}$ and

$$
\eta= \begin{cases}1, & \theta_{1} \in[0,1] \\ \sqrt{1-\left(\left|\theta_{1}\right| / d\right)^{2}} & \theta_{1} \in(-1,0)\end{cases}
$$

Under the hypothesis of the theorem we have $\left|\theta_{1}\right| / d \in(0,1)$ if $\theta_{1} \in(-1,0)$. Then following lemma holds true. 
LEMMA 2.6 Under the hypothesis of Theorem 2.5, there is $k_{0} \in \mathbb{N}$ such that for all $\lambda \in K$ with $|\lambda| \geq k_{0}$ we have

$$
\left\|\left(L_{0}+\delta_{b} L+\lambda^{2} C\right) u\right\|_{-} \geq\left(\eta-\left\|\delta_{b} L\right\|\right)\|u\|_{+}
$$

for all $u \in H^{+}(D)$ and $\lambda \in K$, and there are positive constants $p_{1}=p_{1}(K), q_{1}=$ $q_{1}(K)$ such that

$$
\left\|\left(L_{0}+\delta_{b} L+\lambda^{2} C\right) u\right\|_{-} \geq p_{1}\|u\|_{+}+q_{1}|\lambda|^{2}\|C u\|_{-}
$$

for all $u \in H^{+}(D)$ and $\lambda \in K$.

Proof. Given any $u \in H^{+}(D)$, an easy computation with the use of formulas (8) and (9) shows that

$$
\begin{gathered}
\lambda^{2}\langle C u, u\rangle=|\lambda|^{2} \int_{D}\left|a_{0}^{(2)}(z) \| u(z)\right|^{2} e^{\sqrt{-1}\left(\varphi_{0}(z)+2 \varphi_{\lambda}\right)} d x, \\
\left\|\left(L_{0}+\lambda^{2} C\right) u\right\|_{-}^{2}=\left\langle u+\lambda^{2} L_{0}^{-1} C u,\left(L_{0}+\lambda^{2} C\right) u\right\rangle^{2}= \\
\left\langle u, L_{0} u\right\rangle+\left\langle\lambda^{2} L_{0}^{-1} C u, \lambda^{2} C u\right\rangle+\bar{\lambda}^{2}\langle u, C u\rangle+\lambda^{2}\left\langle L_{0}^{-1} C u, L_{0} u\right\rangle= \\
\|u\|_{+}^{2}+|\lambda|^{4}\|C u\|_{-}^{2}+\bar{\lambda}^{2}\langle u, C u\rangle+\lambda^{2}\left(L_{0}^{-1} C u, u\right)_{+}= \\
\|u\|_{+}^{2}+|\lambda|^{4}\|C u\|_{-}^{2}+\bar{\lambda}^{2}\langle u, C u\rangle+\lambda^{2}\langle C u, u\rangle= \\
\|u\|_{+}^{2}+|\lambda|^{4}\|C u\|_{-}^{2}+2 \Re\left(\lambda^{2}\langle C u, u\rangle\right) .
\end{gathered}
$$

For all $\lambda \in K$ we have

$$
\Re\left(\lambda^{2}\langle C u, u\rangle\right)=|\lambda|^{2} \int_{D}\left|a_{0}^{(2)}(z) \| u(z)\right|^{2} \cos \left(\varphi_{0}(z)+2 \varphi_{\lambda}\right) d x .
$$

If $\theta_{1} \in[0,1]$ then $\eta=1$ and for all $u \in H^{+}(D)$ we have:

$$
\begin{gathered}
\left\|\left(L_{0}+\lambda^{2} C\right) u\right\|_{-}^{2} \geq\|u\|_{+}^{2}+|\lambda|^{4}\|C u\|_{-}^{2} \\
\left\|\left(L_{0}+\delta_{b} L+\lambda^{2} C\right) u\right\|_{-} \geq\left\|\left(L_{0}+\lambda^{2} C\right) u\right\|_{-}\left\|\delta_{b} L u\right\|_{-} \geq \\
\sqrt{\|u\|_{+}^{2}+|\lambda|^{4}\|C u\|_{-}^{2}}-\left\|\delta_{b} L u\right\|_{-} .
\end{gathered}
$$


Clearly, for $\alpha \in[0, \pi / 2]$ and non-negative numbers $a, b$ we have

$$
\sqrt{a+b} \geq \sqrt{a} \cos (\alpha)+\sqrt{b} \sin (\alpha)
$$

As $\left\|\delta_{b} L\right\|<\eta=1$, there is $\alpha_{0} \in(0, \pi / 2)$ such that

$$
\left\|\delta_{b} L\right\|<\cos \left(\alpha_{0}\right)
$$

In particular, this means that for all $u \in H^{+}(D)$ and all $\lambda \in K$ we have

$$
\begin{gathered}
\left\|\left(L_{0}+\delta_{b} L+\lambda^{2} C\right) u\right\|_{-} \geq\|u\|_{+}-\left\|\delta_{b} L u\right\|_{-} \geq\left(1-\left\|\delta_{b} L\right\|\right)\|u\|_{+}, \\
\left\|\left(L_{0}+\delta_{b} L+\lambda^{2} C\right) u\right\|_{-} \geq \cos \left(\alpha_{0}\right)\|u\|_{+}+\sin \left(\alpha_{0}\right)|\lambda|^{2}\|C u\|_{-}-\left\|\delta_{b} L u\right\|_{-} \geq \\
\left(\cos \left(\alpha_{0}\right)-\left\|\delta_{b} L\right\|\right)\|u\|_{+}+\sin \left(\alpha_{0}\right)|\lambda|^{2}\|C u\|_{-},
\end{gathered}
$$

i.e. the desired inequalities are true if $\theta_{1} \in[0,1]$.

If $\theta_{1} \in(-1,0)$ then, by $(23)$ and $(17)$,

$$
\Re\left(\lambda^{2}\langle C u, u\rangle\right) \geq-\left|\theta_{1}\right||\lambda|^{2} \int_{D}\left|a_{0}^{(2)}(z)\right||u(z)|^{2} d x .
$$

Let us prove that for any $\theta \in\left(\left|\theta_{1}\right| / d, 1\right]$ and $\gamma \in[0,1]$ with $\theta \sqrt{1-\gamma}>\left|\theta_{1}\right| / d$ there is $k_{0} \in \mathbb{N}$ such that

$$
\left\|\left(L_{0}+\lambda^{2} C\right) u\right\|^{2} \geq\left(1-\theta^{2}\right)\|u\|_{+}^{2}+\gamma|\lambda|^{4}\|C u\|_{-}^{2}
$$

for all $u \in H^{+}(D)$ and all $\lambda \in K$ with $|\lambda| \geq k_{0}$.

Indeed, we argue by contradiction. Let there are $\theta \in\left(\left|\theta_{1}\right| / d, 1\right]$ and $\gamma \in[0,1)$ with $\theta \sqrt{1-\gamma}>\left|\theta_{1}\right| / d$ such that for each $k \in \mathbb{N}$ there are $u_{k} \in H^{+}(D)$ with $\left\|u_{k}\right\|_{+}=1$, and a number $\lambda_{k} \in K$ with $\left|\lambda_{k}\right| \geq k$ such that

$$
\left\|\left(L_{0}+\lambda_{k}^{2} C\right) u_{k}\right\|^{2}<1-\theta^{2}+\gamma\left|\lambda_{k}\right|^{4}\left\|C u_{k}\right\|_{-}^{2} .
$$

It follows from (22) and (23) that

$$
\theta^{2}+\left|\lambda_{k}\right|^{4}\left\|C u_{k}\right\|_{-}^{2}(1-\gamma)+2\left|\lambda_{k}\right|^{2} \int_{D} \cos \left(\varphi_{0}+2 \varphi_{\lambda_{k}}\right)\left|a_{0}^{(2)}(z)\right|\left|u_{k}(z)\right|^{2} d x<0,
$$

i.e.

$$
\begin{gathered}
\left(\theta-\sqrt{(1-\gamma)}\left|\lambda_{k}\right|^{2}\left\|C u_{k}\right\|_{-}\right)^{2}+ \\
2\left(\theta \sqrt{(1-\gamma)}+\frac{\int_{D} \cos \left(\varphi_{0}+2 \varphi_{\lambda_{k}}\right)\left|a_{0}^{(2)}(z) \| u_{k}(z)\right|^{2} d x}{\left\|C u_{k}\right\|_{-}}\right)\left|\lambda_{k}\right|^{2}\left\|C u_{k}\right\|_{-}<0,
\end{gathered}
$$


for all $k \in \mathbb{N}$.

On the other hand, for all $u \in H^{+}(D)$ with $\|u\|_{+}=1$ we have

$$
\|C u\|_{-}=\left\|e^{2 \sqrt{-1} \varphi_{\lambda_{k}}} C u\right\|_{-} \geq\left|\left(e^{\sqrt{-1}\left(\varphi_{0}+2 \varphi_{\lambda_{k}}\right)}\left|a_{0}^{(2)}\right| u, u\right)_{L^{2}(D)}\right| .
$$

In particular, we have

$$
\left|\frac{\int_{D} \cos \left(\varphi_{0}+\varphi_{\lambda_{k}}\right)\left|a_{0}^{(2)}(z) \| u_{k}(z)\right|^{2} d x}{\left\|C u_{k}\right\|_{-}}\right| \leq 1 \text { for all } k \in \mathbb{N} .
$$

Now, if the sequence $\left\{\left|\lambda_{k}\right|^{2}\left\|C u_{k}\right\|_{-}\right\}$is unbounded then extracting a subsequence $\left\{\left|\lambda_{k_{j}}\right|^{2}\left\|C u_{k_{j}}\right\|_{-}\right\}$tending to $+\infty$, dividing (27) by $\left|\lambda_{k_{j}}\right|^{4}\left\|C u_{k_{j}}\right\|_{-}^{2}$ and passing to the limit with respect to $k_{j} \rightarrow+\infty$ we obtain $1 \leq 0$, a contradiction.

Let the sequence $\left\{\left|\lambda_{k}\right|^{2}\left\|C u_{k}\right\|_{-}\right\}$be bounded. As $L_{0}$ is bounded it maps the bounded sequence $\left\{u_{k}\right\}$ to the bounded sequence $\left\{L_{0} u_{k}\right\}$. Now the weak compactness principle for Hilbert spaces yields that there is a subsequence $\left\{u_{k_{j}}\right\}$ weakly convergent to an element $u_{0}$ in the space $H^{+}(D)$. Then $\left\{C u_{k_{j}}\right\}$ converges to $C u_{0}$ in $H^{-}(D)$ because $C: H^{+}(D) \rightarrow H^{-}(D)$ is compact and $\left\{u_{k_{j}}\right\}$ converges to $u_{0}$ in $L^{2}(D)$ because the embedding $\iota: H^{+}(D) \rightarrow L^{2}(D)$ is compact, too. Since the sequence $\left\{\lambda_{k_{j}}^{2} C u_{k_{j}}\right\}$ is bounded in $H^{-}(D)$ and $\left|\lambda_{k}\right| \rightarrow+\infty$ we conclude that $\left\{C u_{k_{j}}\right\}$ converges to zero in $H^{-}(D)$. This means that $C u_{0}=0$ and then $u_{0}=0$ because the operator $C$ is injective.

According to compactness principle, we may consider the subsequences

$$
\left\{\left|\lambda_{k_{j}}\right|^{2}\left\|C u_{k_{j}}\right\|_{-}\right\} \text {and }\left\{-\frac{\int_{D} \cos \left(\varphi_{0}+2 \varphi_{\lambda_{k_{j}}}\right)\left|a_{0}^{(2)}(z)\right|\left|u_{k_{j}}(z)\right|^{2} d x}{\left\|C u_{k_{j}}\right\|_{-}}\right\}
$$

as convergent to the limits $\alpha \geq 0$ and $\beta \in[-1,1]$ respectively. Now it follows from (27) that

$$
(\theta-\alpha)^{2}+2 \alpha(\theta-\beta) \leq 0
$$

If $\alpha=0$ then we have a contradiction because $\theta>0$. If $\alpha>0$ and $\beta \leq 0$ then $\theta-\beta>0$ and we again have a contradiction.

Let $\alpha>0$ and $\beta>0$. Since $u e^{\sqrt{-1} \varphi_{0,0}} \in H^{+}(D)$ we see that

$$
\begin{gathered}
\|C u\|_{-} \geq \frac{\left|\left(e^{\sqrt{-1}\left(\varphi_{0}-\varphi_{0,0}\right)}\left|a_{0}^{(2)}\right| u, u\right)_{L^{2}(D)}\right|}{\left\|u e^{\sqrt{-1} \varphi_{0,0}}\right\|_{+}}= \\
\frac{\left|\left(\left(1-\left(1-e^{\sqrt{-1}\left(\varphi_{0}-\varphi_{0,0}\right)}\right)\right)\left|a_{0}^{(2)}\right| u, u\right)_{L^{2}(D)}\right|}{\left\|u e^{\sqrt{-1} \varphi_{0,0}}\right\|_{+}} \geq \\
\frac{\left(1-\left\|1-e^{\sqrt{-1}\left(\varphi_{0}-\varphi_{0,0}\right)}\right\|_{L^{\infty}(D)}\right)\left(\left|a_{0}^{(2)}\right| u, u\right)_{L^{2}(D)}}{\left\|u e^{\sqrt{-1} \varphi_{0,0}}\right\|_{+}} .
\end{gathered}
$$


Hence if $\left\|1-e^{\sqrt{-1}\left(\varphi_{0}-\varphi_{0,0}\right)}\right\|_{L^{\infty}(D)}<1$ then

$$
\limsup _{k_{j} \rightarrow \infty} \frac{\left(\left|a_{0}^{(2)}\right| u_{k_{j}}, u_{k_{j}}\right)_{L^{2}(D)}}{\left\|C u_{k_{j}}\right\|_{-}} \leq \frac{\lim \sup _{k_{j} \rightarrow \infty}\left\|u_{k_{j}} e^{\sqrt{-1} \varphi_{0,0}}\right\|_{+}}{1-\left\|1-e^{\sqrt{-1}\left(\varphi_{0}-\varphi_{0,0}\right)}\right\|_{L^{\infty}(D)}} .
$$

On the other hand, as $\left|e^{\sqrt{-1} \varphi_{0,0}(x)}\right|=1$ we conclude that

$$
\begin{gathered}
\left\|u e^{\sqrt{-1} \varphi_{0,0}}\right\|_{+}^{2}=\|u\|_{+}^{2}+\sum_{j, k=1}^{n}\left(\left(\bar{\partial}_{j} e^{\sqrt{-1} \varphi_{0,0}}\right) u,\left(\bar{\partial}_{k} e^{\sqrt{-1} \varphi_{0,0}}\right) u\right)_{L^{2}(D)}+ \\
2 \Re\left(\sum_{j, k=1}^{n}\left(\left(\bar{\partial}_{j} e^{\sqrt{-1} \varphi_{0,0}}\right) u, e^{\sqrt{-1} \varphi_{0,0}} \bar{\partial}_{k} u\right)_{L^{2}(D)}\right)
\end{gathered}
$$

for all $u \in H^{+}(D)$.

Then, as $u_{k_{j}} \rightarrow 0$ in $L^{2}(D)$ and $\left\|u_{k_{j}}\right\|_{+}=1$, it follows from (29) that

$$
\limsup _{k_{j} \rightarrow \infty}\left\|u_{k_{j}} e^{\sqrt{-1} \varphi_{0,0}}\right\|_{+}=1 \text { for all } j \in \mathbb{N} \text {. }
$$

Therefore, if $\beta>0$ then, by (25),

$$
\begin{gathered}
\beta=\lim _{k_{j} \rightarrow \infty} \frac{-\int_{D} \cos \left(\varphi_{0}+2 \varphi_{\lambda_{k_{j}}}\right)\left|a_{0}^{(2)}(z) \| u_{k_{j}}(z)\right|^{2} d x}{\left\|C u_{k_{j}}\right\|_{-}} \leq \\
\quad \limsup _{k_{j} \rightarrow \infty}\left|\theta_{1}\right| \frac{\int_{D}\left|a_{0}^{(2)}(z) \| u_{k_{j}}(z)\right|^{2} d x}{\left\|C u_{k_{j}}\right\|_{-}} \leq \frac{\left|\theta_{1}\right|}{d} .
\end{gathered}
$$

This means that $\theta-\beta>0$ if $\theta>\left|\theta_{1}\right| / d$ and we again have a contradiction with (28). Thus, (26) is fulfilled.

Finally, as $\left\|\delta_{b} L\right\|^{2}<1-\left(\left|\theta_{1}\right| / d\right)^{2}$ we see that there are $\gamma_{0} \in\left[0,1-\left(\left|\theta_{1}\right| / d\right)^{2}\right)$ and $\alpha_{1} \in(0, \pi / 2)$ such that

$$
\left\|\delta_{b} L\right\|<\cos \left(\alpha_{1}\right)\left(1-\frac{\left|\theta_{1}\right|^{2}}{\left(1-\gamma_{0}\right) d^{2}}\right)^{1 / 2} .
$$

Therefore, using (24), (26) we see that

$$
\begin{gathered}
\left\|\left(L_{0}+\delta_{b} L+\lambda^{2} C\right) u\right\|_{-} \geq \sqrt{\left(1-\frac{\left|\theta_{1}\right|^{2}}{\left(1-\gamma_{0}\right) d^{2}}\right)\|u\|_{+}^{2}+\gamma_{0}|\lambda|^{4}\|C u\|_{-}^{2}}-\left\|\delta_{b} L u\right\|_{-} \geq \\
\cos \left(\alpha_{1}\right)\left(1-\frac{\left|\theta_{1}\right|^{2}}{\left(1-\gamma_{0}\right) d^{2}}\right)^{1 / 2}\|u\|_{+}+\sin \left(\alpha_{1}\right) \sqrt{\gamma_{0}}|\lambda|^{2}\|C u\|_{-}-\left\|\delta_{b} L u\right\|_{-} \geq
\end{gathered}
$$




$$
\left(\cos \left(\alpha_{1}\right)\left(1-\frac{\left|\theta_{1}\right|^{2}}{\left(1-\gamma_{0}\right) d^{2}}\right)^{1 / 2}-\left\|\delta_{b} L\right\|\right)\|u\|_{+}+\sin \left(\alpha_{1}\right) \sqrt{\gamma_{0}}|\lambda|^{2}\|C u\|_{-} .
$$

for all $u \in H^{+}(D)$ and all $\lambda \in K$.

Further, using by Lemma 2.6 and arguments, analogous [14, Theorem 3.8, p.3322] or $[15$, Theorem 3, p.7] we obtain the statement of the theorem.

Note that inequality (19) actually means that the function $a_{0}^{(2)}(z)$ can have the discontinuous argument $\varphi_{0}(z)$.

\section{Spectral properties of the problem}

Suppose $\lambda_{0} \in \mathbb{C}$ and $F(\lambda)$ is a holomorphic function in a punctured neighbourhood of $\lambda_{0}$ which takes on its values in the space $\mathcal{L}\left(H_{1}, H_{2}\right)$ of bounded linear operators, acting from $H_{1}$ in $H_{2}$. The point $\lambda_{0}$ is called a characteristic point of $F(\lambda)$ if there exists a holomorphic function $u(\lambda)$ in a neighborhood of $\lambda_{0}$ with values in $H_{1}$, such that $u\left(\lambda_{0}\right) \neq 0$ but $F(\lambda) u(\lambda)$ extends to a holomorphic function near $\lambda_{0}$ and vanishes at this point. We call $u(\lambda)$ a root function of $F(\lambda)$ at $\lambda_{0}$.

Denote by $T(\lambda)$ the unbounded linear operator $H^{+}(D) \rightarrow H^{-}(D)$ with domain $D_{T(\lambda)}=H^{+}(D)$ which maps an element $u \in D_{T(\lambda)}$ to $L(\lambda) u$. For each $\lambda$ the operator $T(\lambda)$ is clearly closed under assumptions of Lemma 2.2 , because of inequality

$$
\|u\|_{+} \leq\left(\|L(\lambda) u\|_{-}+\|u\|_{-}\right) .
$$

It is densely defined as $H^{1}(D, S) \subset H^{+}(D)$ is dense in $H^{-}(D)$. As well known the null space of $T(\lambda)$ is finite dimensional in $H^{+}(D)$ and its range is closed in $H^{-}(D)$.

In order to define root elements of the family $T(\lambda)$, we assume that there is at least one point $\gamma_{0}$ where $L\left(\gamma_{0}\right)$ is continuously invertible. Then, according to our assumption,

$$
T(\lambda)=T\left(\gamma_{0}\right)\left(I+\left(\lambda^{2}-\gamma_{0}^{2}\right) L^{-1}\left(\gamma_{0}\right) C\right) \text { on } D_{T(\lambda)}=H^{+}(D) .
$$

As the operator $L^{-1}\left(\gamma_{0}\right)$ is continuously invertible, the family

$$
\left\{F_{0}(\lambda)=\left(I+\left(\lambda^{2}-\gamma_{0}^{2}\right) L^{-1}\left(\gamma_{0}\right) C\right)\right\}
$$

consists of Fredholm bounded operators in the space $H^{+}(D)$.

Since the operator $T\left(\gamma_{0}\right)$ is injective, it is natural to call the characteristic values and the root vectors of the family $\left\{F_{0}(\lambda): H^{+}(D) \rightarrow H^{+}(D)\right\}$ of bounded operators by the characteristic values and the root vectors of the family of the closed operators $\left\{T(\lambda): H^{-}(D) \rightarrow H^{-}(D)\right\}$, respectively (see [15]).

Clearly, if $\lambda \neq \gamma_{0}$ then $F_{0}(\lambda) u(\lambda)=0$ if and only if

$$
\left(L^{-1}\left(\gamma_{0}\right) C-\frac{I}{\gamma_{0}^{2}-\lambda^{2}}\right) u(\lambda)=0 .
$$

Therefore the set of the root vectors of the family $\left\{F_{0}(\lambda)\right\}$ coincides with the set of the root vectors of the compact operator $L^{-1}\left(\gamma_{0}\right) C$. 
To formulate the completeness results regarding to parameter-dependent elliptic operators, we note that, under (18), the Hermitian form

$$
h_{0}(u, v)=\left(\left|a_{0}^{(2)}\right| u, v\right)_{L^{2}(D)}
$$

defines a scalar product on $L^{2}(D)$ such that the corresponding norm is equivalent to the original norm of this space.

THEOREM 3.1 Let (5) hold and $\delta b_{0}=0, \delta a_{0}=0$ and $a_{k}=0$ for all $1 \leq k \leq n$. If (18) is fulfilled and $\varphi_{0}(z)=\varphi_{0,0}$ for all $z \in \bar{D}$ with a constant $\varphi_{0,0} \in[0,2 \pi)$ then

1) the operators $T(\lambda)$ are continuously invertible for all $\lambda \in \mathbb{C}$ except a countable number of the characteristic values $\left\{\lambda_{\nu}\right\}$ where each $\lambda_{\nu}$ belongs to one of the rays $\left\{\arg (\lambda)=\left(\varphi_{0,0} \pm \pi\right) / 2\right\}$ and $\lim _{\nu \rightarrow \infty}\left|\lambda_{\nu}\right|=+\infty$;

3) the root vectors $\left\{b_{\nu}\right\}$ of the family $\{T(\lambda)\}$ are complete in the spaces $H^{+}(D)$, $L^{2}(D)$ and $H^{-}(D)$;

4) the system $\left\{b_{\nu}\right\}$ is orthogonal bases in $H^{+}(D)$ and in the space $L^{2}(D)$ with the scalar product $h_{0}(\cdot, \cdot)$; moreover the system $\left\{\sqrt{\left|a_{0}^{(2)}\right|} b_{\nu}\right\}$ is orthogonal basis in $L^{2}(D)$ and the system $\left\{\left|a_{0}^{(2)}\right| b_{\nu}\right\}$ is an orthogonal basis in $H^{-}(D)$.

Proof. Denote by $\tilde{C}: H^{+}(D) \rightarrow H^{-}(D)$ the operator that is induced by the term $\left|a_{0}^{(2)}(x)\right|$. The proof of the theorem is based on the following expectable lemma.

Lemma 3.2 Let the hypothesis of Lemma 2.2 and (18) hold true. Then the operator $L_{0}^{-1} \tilde{C}: H^{+}(D) \rightarrow H^{+}(D)$ is compact, self-adjoint and its order is finite:

$$
\operatorname{ord}\left(L_{0}^{-1} \tilde{C}\right)=n \text {. }
$$

Proof. Under the hypothesis of Lemma 2.2 the space $H^{+}(D)$ is continuously embedded to $H^{1 / 2-\varepsilon}(D)$ with any $\varepsilon>0$. Then, according to Rellich Theorem, the embedding $\iota: H^{+}(D) \rightarrow L^{2}(D)$ is compact. Denote by $\iota^{\prime}: L^{2}(D) \rightarrow H^{-}(D)$ the adjoint to $\iota$ with respect to the pairing $\langle\cdot, \cdot\rangle$.

Note that the function $\left|a_{0}^{(2)}\right| \in L^{\infty}(D)$ induces a bounded self-adjoint operator $C_{0}: L^{2}(D) \rightarrow L^{2}(D)$. Then

$$
L_{0}^{-1} \tilde{C}=L_{0}^{-1} \iota^{\prime} C_{0} \iota
$$

and hence the operator $L_{0}^{-1} \tilde{C}$ is compact. Moreover,

$$
\begin{gathered}
\left(L_{0}^{-1} \tilde{C} u, v\right)_{+}=<\iota^{\prime} C_{0} \iota u, v>=\left(C_{0} \iota u, \iota v\right)_{L^{2}(D)}=\int_{D}\left|a_{0}^{(2)}(z)\right| u(z) \bar{v}(z) d x \\
\left(u, L_{0}^{-1} \tilde{C} v\right)_{+}=\overline{\left(L_{0}^{-1} C_{0} v, u\right)_{+}}=\overline{\left(C_{0} \iota v, \iota u\right)_{L^{2}(D)}}=\int_{D}\left|a_{0}^{(2)}(z)\right| u(z) \bar{v}(z) d x
\end{gathered}
$$

for all $u, v \in H^{+}(D)$, i.e. the operator $L_{0}^{-1} \tilde{C}$ is self-adjoint.

As the operator $\iota$ is injective, we see that

$$
\left(L_{0}^{-1} \iota^{\prime} C_{0} \iota-\mu I\right) u=0
$$


if and only if

$$
\left(\iota L_{0}^{-1} \iota^{\prime} C_{0}-\mu I\right) \iota u=0 .
$$

Therefore

$$
\left(L_{0}^{-1} \iota^{\prime} C_{0} \iota-\mu I\right)^{m} u=0
$$

with some $m \in \mathbb{N}$ if and only if

$$
\left(\iota L_{0}^{-1} \iota^{\prime} C_{0}-\mu I\right)^{m} \iota u=0 .
$$

Thus, the set of eigen-values of the operator $L_{0}^{-1} \tilde{C}$ coincides with the set of eigenvalues of the operator $\iota L_{0}^{-1} \iota^{\prime} C_{0}$. Besides the multiplicities of the eigen-values coincide, too.

According to [14, Corollary 3.5], the operator $\iota L_{0}^{-1} \iota^{\prime}: L^{2}(D) \rightarrow L^{2}(D)$ is compact self-adjoint and its order is finite:

$$
\operatorname{ord}\left(\iota L_{0}^{-1} \iota^{\prime}\right)=n \text {. }
$$

As $C_{0}: L^{2}(D) \rightarrow L^{2}(D)$ is bounded, the operators $\iota L_{0}^{-1} \iota^{\prime}$ and $\iota L_{0}^{-1} \iota^{\prime} C_{0}$ have the same orders (see [4, Ch. 2, § 2], [3] or elsewhere). By the discussion above, the orders of the operators $\iota L_{0}^{-1} \iota^{\prime} C_{0}$ and $L_{0}^{-1} \tilde{C}$ coincide.

Let us continue the proof of the theorem. As $\delta b_{0}=0, \delta a_{0}=0, a_{k}=0$ for all $1 \leq k \leq n$ and the operator $L_{0}$ is continuously invertible, we may take $\gamma_{0}=0$. Then (30) yields

$$
T(\lambda)=\lambda^{2} e^{\sqrt{-1} \varphi_{0,0}} L_{0}\left(L_{0}^{-1} \tilde{C}-\lambda^{-2} e^{\sqrt{-1}\left(\pi-\varphi_{0,0}\right)} I\right) \text { on } D_{T(\lambda)}=H^{+}(D) .
$$

According to Lemma 3.2, operator $L_{0}^{-1} \tilde{C}$ is compact self-adjoint and

$$
\operatorname{ord}\left(L_{0}^{-1} \tilde{C}\right)=n \text {. }
$$

By the Hilbert-Schmidt Theorem, there is an orthonormal basis $\left\{b_{\nu}\right\}$ in $H^{+}(D)$, consisting of the eigen-vectors of the operator $L_{0}^{-1} \tilde{C}$. Moreover, using (31) we see that

$$
h_{0}\left(b_{\nu}, b_{k}\right)=\left(\sqrt{\left|a_{0}^{(2)}\right|} b_{\nu}, \sqrt{\left|a_{0}^{(2)}\right|} b_{k}\right)_{L^{2}(D)}=\left(L_{0}^{-1} \tilde{C} b_{\nu}, b_{k}\right)_{+}=\mu_{\nu} \delta_{\nu, k}
$$

where $\delta_{\nu, k}$ is the Kronecker symbol. Hence, as $\mu_{\nu}>0$, we see that $\left\{b_{\nu}\right\}$ is orthogonal with respect to $h_{0}(\cdot, \cdot)$ and $\left\{\sqrt{\left|a_{0}^{(2)}\right|} b_{\nu}\right\}$ is orthogonal in $L^{2}(D)$. It is complete in $L^{2}(D)$ because $H^{+}(D)$ is dense in $L^{2}(D)$. Moreover, by the construction, the space $H^{+}(D)$ is dense in $H^{-}(D)$ and hence the system $\left\{b_{\nu}\right\}$ is complete $H^{-}(D)$. Finally,

$$
\left(\left|a_{0}^{(2)}\right| b_{\nu},\left|a_{0}^{(2)}\right| b_{k}\right)_{-}=\left(L_{0}^{-1} \tilde{C} b_{\nu}, L_{0}^{-1} \tilde{C} b_{k}\right)_{+}=\mu_{\nu} \mu_{k} \delta_{\nu, k}
$$


i.e. $\left\{\left|a_{0}^{(2)}\right| b_{\nu}\right\}$ is orthogonal in $H^{-}(D)$. It is complete because

$$
L_{0}^{-1} u=\sum_{\nu}\left(L_{0}^{-1} u, b_{\nu}\right)_{+} b_{\nu}
$$

for each $u \in H^{-}(D)$ by the discussion above and then

$$
u=\sum_{\nu}\left(L_{0}^{-1} u, b_{\nu}\right)_{+} L_{0} b_{\nu}=\sum_{\nu} \frac{\left(L_{0}^{-1} u, b_{\nu}\right)_{+}}{\mu_{\nu}} \tilde{C} b_{\nu}
$$

hence the system $\left\{\left|a_{0}^{(2)}\right| b_{\nu}\right\}$ is an orthogonal basis in $H^{-}(D)$.

As $\mu_{\nu}=\lambda_{\nu}^{-2} e^{\sqrt{-1}\left(\pi-\varphi_{0,0}\right)}>0$, we conclude that the characteristic values $\lambda_{\nu}=$ $\pm \mu_{\nu}^{-1 / 2} e^{\sqrt{-1}\left(\varphi_{0,0}-\pi\right) / 2}$ of the family $T(\lambda)$ lie on one of the rays $\left\{\arg (\lambda)=\left(\varphi_{0,0} \pm\right.\right.$ $\pi) / 2\}$, respectively, and $\lim _{\nu \rightarrow \infty}\left|\lambda_{\nu}\right|=+\infty$.

Now we can use the Keldysh' Theorem about perturbation of compact self-adjoint operators (see [5]).

COROllary 3.3 Let the hypothesis of Lemma 2.2 hold true and $\delta b_{0}=0$. If (18) is fulfilled and $\varphi_{0}(z)=\varphi_{0,0}$ for all $z \in \bar{D}$ with a constant $\varphi_{0,0} \in[0,2 \pi)$ then

1) the operators $T(\lambda)$ are continuously invertible for all $\lambda \in \mathbb{C}$ except a countable number of the characteristic values $\left\{\lambda_{\nu}\right\}$;

2) $\lim _{\nu \rightarrow \infty}\left|\lambda_{\nu}\right|=+\infty$ and for any $\varepsilon>0$ all the characteristic values $\lambda_{\nu}$ (except for a finite number) belong to the corners

$$
\left\{\left|\arg (\lambda)-\left(\varphi_{0,0} \pm \pi\right) / 2\right|<\varepsilon\right\}
$$

3) the system of its root vectors is complete in the spaces $H^{+}(D), H^{-}(D)$ and $L^{2}(D)$.

Proof. First all we note that under the hypothesis of the theorem there is $\gamma_{0}$ such that $L\left(\gamma_{0}\right)$ is continuously invertible. Indeed, we can take $\gamma_{0}$ such that $\left\{\arg \left(\gamma_{0}\right)=\right.$ $\left.-\varphi_{0,0} / 2\right\}$, then in this case $\theta_{1}=1$ and Theorem 2.5 implies that for sufficient large $\left|\gamma_{0}\right|$ the operator $L\left(\gamma_{0}\right)$ is continuously invertible. In particular, the operators $L(\lambda)$ and $T(\lambda)$ are continuously invertible for all $\lambda \in \mathbb{C}$ except a countable number of the characteristic values $\left\{\lambda_{\nu}\right\}$;

On the other hand, as $\varphi_{0}(z)=\varphi_{0,0}$ for all $z \in \bar{D}$ with a constant $\varphi_{0,0} \in[0,2 \pi)$ then (30) yields

$$
T(\lambda)=\left(\gamma_{0}^{2}-\lambda^{2}\right) e^{\sqrt{-1} \varphi_{0,0}} L\left(\gamma_{0}\right)\left(L^{-1}\left(\gamma_{0}\right) \tilde{C}-\left(\gamma_{0}^{2}-\lambda^{2}\right)^{-1} e^{-\sqrt{-1} \varphi_{0,0}} I\right) \text { on } H^{+}(D) .
$$

where, as before, the operator $\tilde{C}: H^{+}(D) \rightarrow H^{-}(D)$ is induced by the term $\left|a_{0}^{(2)}(x)\right|$. Thus, according to (30), the proof of the statements 2) and 3) of the theorem can be reduced to the investigation of the properties of the compact operator $L^{-1}\left(\gamma_{0}\right) \tilde{C}$.

Under the hypothesis of the theorem the operator $\delta L_{0}=\left(L\left(\gamma_{0}\right)-L_{0}\right)$ is compact and then the operator $L_{0}^{-1} \delta L_{0} L^{-1}\left(\gamma_{0}\right)$ is compact, too.

On the other hand, easily we obtain

$$
L^{-1}\left(\gamma_{0}\right)-L_{0}^{-1}=-L^{-1}\left(\gamma_{0}\right) \delta L_{0} L_{0}^{-1}
$$


Hence the operator

$$
L^{-1}\left(\gamma_{0}\right) \tilde{C}=L_{0}^{-1} \tilde{C}-L^{-1}\left(\gamma_{0}\right) \delta L_{0} L_{0}^{-1} \tilde{C}
$$

can be considered as a weak perturbation of the self-adjoint operator $L_{0}^{-1} \tilde{C}$.

According to Lemma 3.2 the order of the operator $L_{0}^{-1} \tilde{C}$ is finite. As the operator $L_{0}^{-1} \tilde{C}$ is injective, the statement of the theorem follows from Keldysh' Theorem (see $[5])$.

Using by Theorem about completeness of root functions for operators of highest orders (see [4, Theorem 6.1, Chapter V, Section 6], or elsewhere), we can to perturb operator $L_{0}^{-1} \tilde{C}$ by "small" perturbation. More precisely, we allow to argument $\varphi_{0}(z)$ of function $a_{0}^{(2)}(z)$ to take its values from some bounded closed angle. Namely, the following theorem holds true.

TheOREM 3.4 Under the hypothesis of Theorem 2.5, let

$$
\Phi=\sup _{z, w \in D}\left(\varphi_{0}(z)-\varphi_{0}(w)\right)<\frac{\pi}{n}
$$

then

1) the root vectors $\left\{b_{\nu}\right\}$ of the family $\{T(\lambda)\}$ are complete in the spaces $H^{+}(D)$, $L^{2}(D)$ and $H^{-}(D)$;

2) all characteristic values $\left\{\lambda_{\nu}\right\}$ (except for a finite number) belong to the corners

$$
\left\{\left|\arg (\lambda) \pm \frac{\pi}{2}+\frac{\Phi_{1}+\Phi_{2}}{4}\right|<\frac{\pi}{4 n}+\varepsilon\right\}
$$

for any $\varepsilon>0$, where $\Phi_{1}=\inf _{z \in D} \varphi_{0}(z), \Phi_{2}=\sup _{z \in D} \varphi_{0}(z)$, and moreover $\lim _{\nu \rightarrow \infty}\left|\lambda_{\nu}\right|=$ $+\infty$

Proof. According to conditions of the theorem, there exist a constant $0<\tau<\frac{\pi}{2}$ such that

$$
\Phi=\Phi_{2}-\Phi_{1}=\frac{\pi}{n}-2 \tau
$$

Then the operator $L(\lambda)$ is continuously invertible for sufficiently large $|\lambda|$ in a sector

$$
\frac{-\left(\pi+\Phi_{1}-a \tau\right)}{2} \leq \varphi_{\lambda} \leq \frac{\pi-\Phi_{2}-a \tau}{2}
$$

with arbitrary constant $0<a<1$. Indeed, due to (32) the interval (33) is not empty and in this case we have

$$
\begin{gathered}
\varphi_{0}+2 \varphi_{\lambda} \leq \Phi_{2}+2 \varphi_{\lambda} \leq \Phi_{2}+\pi-\Phi_{2}-a \tau<\pi, \\
\varphi_{0}+2 \varphi_{\lambda} \geq \Phi_{1}+2 \varphi_{\lambda} \geq \Phi_{1}-\pi-\Phi_{1}+a \tau>-\pi .
\end{gathered}
$$

Then under (18), according to Theorem 2.5, there exist a point $\gamma_{0}$ on any ray $-\left(\pi+\Phi_{1}-a \tau\right) / 2 \leq \arg \left(\gamma_{0}\right) \leq\left(\pi-\Phi_{2}-a \tau\right) / 2$ such that the operator $L\left(\gamma_{0}\right)$ is continuously invertible. 
As under (18) the operator $C: H^{+}(D) \rightarrow H^{-}(D)$ is injective, then we have

$$
\left(I-\left(\gamma_{0}^{2}-\lambda^{2}\right) L^{-1}\left(\gamma_{0}\right) C\right) u=0
$$

if and only if

$$
\left(L\left(\gamma_{0}\right) C^{-1}-\left(\gamma_{0}^{2}-\lambda^{2}\right)\right) C u=0 .
$$

Therefore $u$ is a root function of the bounded operator $L^{-1}\left(\gamma_{0}\right) C$ if and only if $C u$ is a root function of the closed operator $L\left(\gamma_{0}\right) C^{-1}$. It means that the investigation of spectral properties of the family of the operators $T(\lambda)$ can be reduced to the investigation of the properties one of the operators $L^{-1}\left(\gamma_{0}\right) C$ and $L\left(\gamma_{0}\right) C^{-1}$.

Note that the multiplication on the function $e^{\sqrt{-1} \varphi_{0}(z)}$ induces a bounded operator $\delta_{C}: L^{2}(D) \rightarrow L^{2}(D)$. By similar arguments as in Lemma 3.2 we see that the order of the operator $L_{0}^{-1} C=L_{0}^{-1} \iota^{\prime} C_{0} \delta_{C} \iota$ equals to $n$ and then the order of the operator $C L^{-1}\left(\gamma_{0}\right): H^{-} \rightarrow H^{-}$is finite and equals to $n$ too. Indeed, the operator $C L^{-1}\left(\gamma_{0}\right)$ can be presented in the following form

$$
C L^{-1}\left(\gamma_{0}\right)=L_{0}\left(L_{0}^{-1} C\right) L^{-1}\left(\gamma_{0}\right)
$$

and hence $C L^{-1}\left(\gamma_{0}\right)$ can be obtained from $L_{0}^{-1} C$ via the multiplication with the bounded operators $L_{0}$ and $L^{-1}\left(\gamma_{0}\right)$, therefore the order of $C L^{-1}\left(\gamma_{0}\right)$ and $L_{0}^{-1} C$ coincide (see, for instance, [4]).

Now we see from (20) that for any $\lambda$ satisfying

$$
-\left(\pi+\Phi_{1}-a \tau\right) \leq 2 \varphi_{\lambda} \leq \pi-\Phi_{2}-a \tau
$$

we have

$$
\left\|\left(L\left(\gamma_{0}\right)-\left(\gamma_{0}^{2}-\lambda^{2}\right) C\right) u\right\|_{-} \geq c_{K}|\lambda|^{2}\|C u\|_{-},
$$

with a constant $c_{K}>0$. Without loss of generality we can take $\gamma_{0}=0$, because the operator $\gamma_{0}^{2} C$ is compact. Then the hypothesis of Theorem 2.5 are fulfilled for the operator

$$
\tilde{L}(\tilde{\lambda})=L_{0}+\delta_{b} L+\tilde{\delta}_{c} L+\tilde{\lambda}^{2} C
$$

too, where $\tilde{\lambda}=\lambda^{2}-\gamma_{0}^{2}$ and $\tilde{\delta}_{c} L=\delta_{c} L+\gamma_{0}^{2} C$. Set $\mu=-\lambda^{2}$. Then for any $\arg (\mu)$ such that

$$
a \tau-\Phi_{1} \leq \arg (\mu) \leq 2 \pi-\Phi_{2}-a \tau
$$

the ray $\{\phi=\arg (\mu)\}$ is a ray of minimal growth of the resolvent of the closed operator $L(0) C^{-1}$ :

$$
\left\|\left(L(0) C^{-1}-\mu\right)^{-1} w\right\|_{-} \leq \frac{c_{K}}{|\mu|}\|w\|_{-} .
$$

According to (34) the angle between any two neighboring rays is less then $\frac{\pi}{n}$. Finally 
we see that

$$
\left(L(0) C^{-1}-\mu\right)^{-1}=\left(I-\mu L^{-1}(0) C\right)^{-1} C L^{-1}(0) .
$$

Then there exist a point $\mu_{0}$ such that the resolvent of the operator $L(0) C^{-1}$ is compact and its order equals to $n$. Then the statement of the theorem follows immediately from [3, Chapter XI, Section 9, Corollary 31, p. 277] (see also [8]).

\section{An example}

Let $D$ be a ball in $\mathbb{C}^{n}$ of radius $R$ with the center at the origin, $D=B(0, R)$. Under the hypothesis of Lemma 2.4, consider the following function $a_{0}^{(2)}(z)$ with the discontinuous argument $\varphi_{0}(z)$,

$$
a_{0}^{(2)}(z)= \begin{cases}|z|, & \text { for all } 0 \leq|z|<\frac{R}{2} \\ |z| e^{\sqrt{-1}\left(\frac{\pi}{n}-2 \tau\right)}, & \text { for all } \frac{R}{2} \leq|z|<R\end{cases}
$$

where $\tau$ is a some constant, $0<\tau<\frac{\pi}{2 n}$. For this function, condition (18) holds true and moreover $\Phi_{1}=0, \Phi_{2}=\frac{\pi}{n}-2 \tau$. Then

$$
\Phi=\sup _{z, w \in D}\left(\varphi_{0}(z)-\varphi_{0}(w)\right)=\frac{\pi}{n}-2 \tau<\frac{\pi}{n} .
$$

As we have seen in the Theorem 3.4, for any $\lambda$ such that $-\left(\pi+\Phi_{1}-a \tau\right) / 2 \leq \varphi_{\lambda} \leq$ $\left(\pi-\Phi_{2}-a \tau\right) / 2$ with some $0<a<1$ we have

$$
\theta_{1}=\min _{z \in D} \cos \left(\varphi_{0}-2 \varphi_{\lambda}\right)=-\cos (a \tau)>-1
$$

Consider now the inequality (19) of Theorem 2.5. For our function $a_{0}^{(2)}(z)$ we have

$$
\begin{array}{r}
I^{2}\left(\varphi_{0,0}\right)=\left\|1-e^{\sqrt{-1}\left(\varphi_{0}-\varphi_{0,0}\right)}\right\|_{L^{\infty}(D)}^{2}=\operatorname{ess} \sup _{z \in D}\left(2-2 \cos \left(\varphi_{0}-\varphi_{0,0}\right)\right) \\
=\operatorname{ess} \sup _{z \in D}\left(2-2 \cos \left(\frac{\pi}{n}-2 \tau-\varphi_{0,0}\right)\right)
\end{array}
$$

It is clear that

$$
\min _{\varphi_{0,0} \in C^{0,1}(\bar{D})} I^{2}\left(\varphi_{0,0}\right)=2-2 \cos \left(\frac{\pi}{2 n}-\tau\right) .
$$

Then (19) takes the following form:

$$
2-2 \cos \left(\frac{\pi}{2 n}-\tau\right)<(1-\cos (a \tau))^{2}
$$

and it follows that there exist a $\tau_{0}$, close to zero, such that condition (19) is not fulfilled. Hence we can not say anything about the rays of minimal growth. Note, that 
if the argument $\varphi_{0}(z)$ of $a_{0}^{(2)}(z)$ is a continuous function, then under the hypothesis of Lemma 2.4, if (18) and some additional restriction on operator $\delta_{b} L$ are fulfilled, [15, Theorem 5] guarantees that the system of root vectors of the family $L(\lambda)$ is complete in the spaces $H^{+}(D), H^{-}(D)$ and $L^{2}(D)$.

\section{Acknowledgement}

The work was supported by the grant of the Russian Federation Government for scientific research under the supervision of leading scientist at the Siberian federal university, contract N. 14.Y26.31.0006.

\section{References}

[1] Agranovich MS, Vishik MI. Elliptic problems with a parameter and parabolic problems of general type. Uspekhi Mat. Nauk. 1964;19:53-161.

[2] Denk R, Volevich L. Parameter-elliptic boundary value problems connected with the Newton polygon. Diff. Int. Eq. 2002;15(3):289-326.

[3] Dunford N, Schwartz JT. Linear Operators, Vol. II, Selfadjoint Operators in Hilbert Space. New York: Intersci. Publ; 1963.

[4] Gokhberg IT, Krein MG. Introduction to the Theory of Linear Nonselfadjoint Operators in Hilbert Spaces. AMS: Providence; 1969.

[5] Keldysh MV. On the characteristic values and characteristic functions of certain classes of non-selfadjoint equations. Dokl. AN SSSR 1951;77:11-14.

[6] Markus AS. Introduction to the Spectral Theory of Polynomial Operator Pencils. Vol. 71. Providence, Rhode Island: Translations of Mathematical Monographs, AMS; 1988.

[7] Yakubov SY. Multiple completeness for systems of operator bundles and elliptic boundary value problem. Matem. Sb. 1990;181:95-113.

[8] Agmon S. On the eigenfunctions and on the eigenvalues of general elliptic boundary value problems. Comm. Pure Appl. Math. 1962;15:119-147.

[9] Browder FE. On the eigenfunctions and eigenvalues of the general elliptic differential operator. Proc. Nat. Acad. Sci. USA. 1953;39:433-439.

[10] Lions JL, Magenes E. Non-Homogeneous Boundary Value Problems und Applications, Vol. 1. Berlin: Springer-Verlag; 1972.

[11] Paltsev BV. Mixed problems with non-homogeneous boundary conditions in Lipschitz domains for second-order elliptic equations with a parameter. Mat. Sb. 1996;187:59116.

[12] Agranovich MS. Spectral Problems in Lipschitz Domains. Modern Mathematics, Fundamental Trends. 2011;39:11-35.

[13] Tarkhanov N. On the root functions of general elliptic boundary value problems. Compl. Anal. Oper. Theory. 2006;1:115-141.

[14] Shlapunov A, Tarkhanov N. On completeness of root functions of Sturm-Liouville problems with discontinuous boundary operators. J. of Differential Equations. 2013;10:3305-3337.

[15] Polkovnikov A, Shlapunov A. On non-coercive mixed problems for parameterdependent elliptic operators. Math. Commun. 2015;20:131-150 